\title{
A Graphical Method for LTE-A System and Digital Broadcasting Interoperability Studies
}

\author{
Zaid Ahmed Shamsan ${ }^{1,2}$ \\ ${ }^{1}$ Faculty of Engineering, Al Imam Mohammad Ibn Saud Islamic University \\ (IMSIU), Riyadh, Saudi Arabia \\ ${ }^{2}$ Faculty of Engineering and Information Technology, Taiz University, Taiz, \\ Yemen \\ shamsan22@gmail.com
}

\begin{abstract}
The co-primary basis operation between long term evolution-advanced (LTE-A) and current digital television (TV) receiver systems is a significant issue when working in cochannel or adjacent channel in the $800 \mathrm{MHz}$. This policy aims to efficient use of frequency spectrum, but leads to an intersystem interference phenomenon, which may disturb the Interoperability between LTE-A and digital TV receiver. In this paper, we propose an effective, simple and graphical approach based on the operating band unwanted emission technique to evaluate all possible intersystem interference scenarios. A variety of practical and substantial spectrum sharing elements that can seriously influence the coexistence situation of LTE-A and TV receiver have been studied. The findings show that co-channel sharing scenario will have much more coexistence difficulty for macro (urban/suburban/rural) areas without employing some interference mitigation techniques. However, in order to make the two systems interoperate in adjacent channel situation, the minimum frequency shift should be at least half of the interferer system bandwidth without mitigation technique. Additionally, the wider channel bandwidth of LTE-A system is preferable more than narrower bandwidth for peaceful compatibility, which helps to support high data rate for LTE-A system.
\end{abstract}

Keywords: LTE-Advanced, spectrum sharing, Radiofrequency interference, Radio propagation, TV broadcasting

\section{Introduction}

In the recent time, various mobile communication providers worldwide have organizing for international mobile telecommunications-advanced (IMT-A) systems, which include long term evolution-advanced (LTE-A) technology. LTE-A is proposed to meet the requirements for IMT-A, according to ITU-R definition [1-3]. LTE-A system is standardized by the $3^{\text {rd }}$ generation partnership project (3GPP) developed from evolveduniversal terrestrial radio access (E-UTRA) series. LTE-A system utilizes carrier aggregation technique to support high bandwidth (BW) up to $100 \mathrm{MHz}$ [4]. This technique is proposed to provide maximum data rates up to $1 \mathrm{Gbps}$ for low speed and 100 Mbps for high speed mobility, respectively. The allocated frequency bands that support these high data rates start from $0.7-3.6 \mathrm{GHz}$ and beyond [5]. With respect to the lower band, 790-862 MHz, it definitely lies within the 'sweet spot', and it is used by a variety of applications; due to its wavelength which is high enough to provide enough coverage with a reasonable base station (BS) number. In addition, it is not susceptible to precipitation attenuation as in case of higher frequencies. Besides, the frequency bandwidths are large and sufficient to support high capacity for serving several communication applications and media services [5]. Consequently, ITU-R has recently decided to be utilized by IMTA systems in the Regions 1 (Europe, Africa and Arab countries) and 3 (Asia). In addition, 
the ITU recommended the $790-862 \mathrm{MHz}$ band to be engaged by a number of dissimilar services including digital broadcasting service, on co-primary basis [6]. This co-primary operation between LTE-A and broadcasting receiver may cause destructive interference between the two services [7, 8] and can affect on the system performance [9-11]. In the current coexistence literature between LTE and digital broadcasting, some studies have been carried out to analyze compatibility between these two systems through ergodic capacity [12], measurements testbed [13-14], and both simulation and measurements [15].

Additionally, in a recent study [16], a method based on the interferer mask and victim receiver blocking techniques is suggested to investigate the intersystem interference between the IMT-A systems and TV system, but to evaluate only three overlapping scenarios. More recently, emission mask is proposed by [17] to explore more dissimilar interference cases between both IMT-Advanced and TV receiver for the macro urban area.

In this paper, a simple and efficient coexistence approach is proposed. It can be applied to the whole spectrum band under consideration to investigate the interoperability conditions and coordinate non co-sited LTE-A downlink and TV receiver systems. The scheme first proposed by [17] is developed and presented in this paper with more details, and it is based on operating band unwanted emissions (OBUE). The OBUE can be used to exactly evaluate all possible intersystem interference overlapping situations: (a) cochannel (full overlapping) scenario, (b) adjacent channel plus an overlapping band $(O B)$ (fractional overlapping) scenario, (c) adjacent channel (with no $O B$ ) scenario and (d) adjacent channel plus protection band $(P B)$ scenario. Generally, different channel bandwidths for the interferer and victim systems, various minimum separation distances required for coexistence, numerous carrier frequency shift and variable protection band size can be managed for both systems under consideration in order to enhance the capability of the LTE-A frequency spectrum sharing. Further, for example, further loss as a result of antenna misalignment is proposed to alleviate the influences of interference on coexistence feasibility. The coexistence studies cover terrestrial macro (urban, suburban and rural) areas, and the relation between system channel bandwidth and both interference scenarios and minimum required frequency shift, which have not been considered in previously presented results [17]. Ultimately, this proposed method aims to facilitate spectrum sharing investigation and suggests possible coordination.

The main contributions of this paper are listed as follows. (1) We model the intersystem interference between LTE-A and digital broadcast downlink system using OBUE through a graphical scheme, (2) we evaluate all possible intersystem interference overlapping situations using different and significant coexistence factors, and (3) we propose some mechanisms to enhance coexistence between LTE-A and digital broadcast systems.

The remainder of the paper is structured as follows. Section 2 introduces the proposed scheme for interoperability evaluation. In sections 3 , system parameters and coexistence conditions are presented in details. The results and distinctive discussion are introduced in section 4. Finally, we conclude the paper in section 5.

\section{Intersystem Interference Operability Method}

Interference between wireless systems occurs when the systems operate at an overlapping frequency band or channels and at the same time share the same terrestrial environment, which leads to losses in terms of capacity and causes coverage limitation. Intersystem interference can be estimated according to the criteria of the permissible interference level at the victim receiver [18]. In this paper, it is assumed that the minimum detected signal power of digital TV receiver is $-116 \mathrm{dBm}$ according to CEPT [19] and FCC [20]. This threshold is assumed due to the fact that the LTE-A system effect will be insignificant when its transmitted power is smaller than the minimum level to a TV receiver. On the other side, if the power level of LTE-A is higher than the minimum level 
and the difference between desired signal and interference signal is not high enough, LTE-A can cause harmful interference on TV receiver. The interference power level, $I$ $(\mathrm{dBm})$ can be expressed by the following equation [6], [17]:

$$
I(\Delta f)=E I R P_{t}+G_{r}+F_{c b w}-P_{\text {loss }}+O B U E_{\text {loss }}(\Delta f)
$$

where $E I R P_{t}(\mathrm{dBm})$ is the effective isotropic radiated power of the transmitter including the gain of antenna and loss due to cable of the interferer (LTE-A), $G_{r}(\mathrm{dBi})$ represents the antenna gain of victim (TV receiver), $F_{c b w}$ is the correction bandwidth factor which depends on both bandwidth of the interferer $\left(B W_{I}\right)$ and victim $(B W v)$ systems, $P_{\text {loss }}$ is the path loss due to signal propagation between the interferer and victim receiver, and $O B U E_{\text {loss }}$ is the LTE-A operating band unwanted emissions represented by spectral emission mask loss due to interferer frequency shift $(\Delta f)$ from carrier frequency.

The OBUE factor of the interferer system is one of the dominant elements that impact on the capability of non co-located systems to operate at the same time with no harmful interference because it restricts the radiated power according to its limitation levels for each $\Delta f$. OBUEs are resulting from the modulation procedure and non linearity in the transmitter except spurious emissions. It consists of the carrier, out of band (OOB) and 10 $\mathrm{MHz}$ on each side to permit higher emission levels at the channel edge due to high transmitted power (see Figure 1).

\subsection{The Proposed Graphical Scheme}

In this spectrum sharing study, LTE OBUE limits are utilized according to [4]. These limits can be converted to linear equations depending on the interferer channel bandwidth such that channel spacing $\left(C h_{\text {spacing }}\right)$ at each $\Delta f$ is given the equivalent power spectrum density (PSD) according to equation (2).

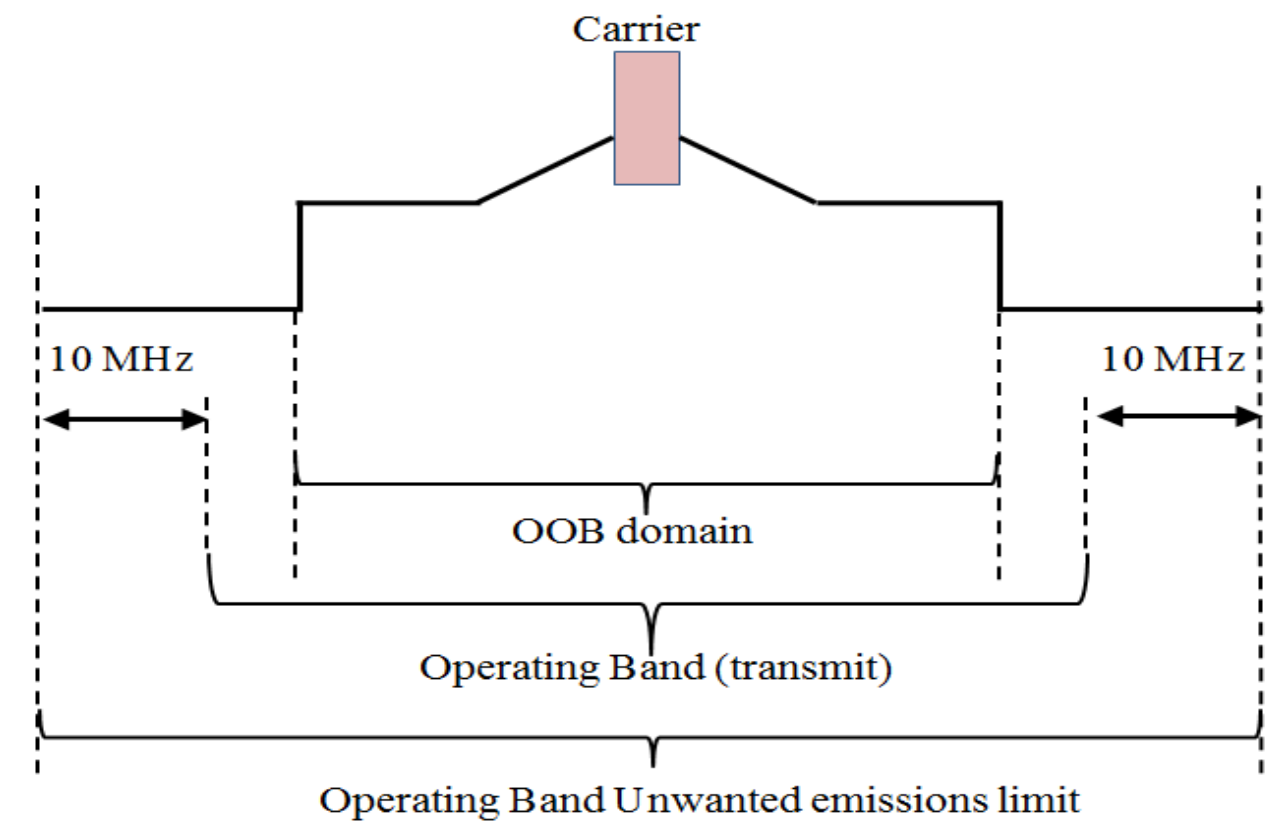

Figure 1. Defined Frequency Ranges For The Operating Band Unwanted
Emissions

$$
C h_{\text {spacing }}=B W_{I} \times \Delta f \text { for } 0 M H z \leq \Delta f \leq 2.5 B W_{I} \mathrm{MHz} \text {. }
$$

The equivalent PSD is treated as attenuation at each frequency shift, OBUE $(\Delta f)$, which participates in the total power link budget calculation from the carrier frequency at $\Delta f=0$ $\mathrm{MHz}$ up to $\Delta f=\left(2.5 \times B W_{I}\right) \mathrm{MHz}$. Consequently, Figure 2 is formed for different interference scenarios. 
Figure 2 consists of $y$-axis which represents the interference power level, and $x$-axis which represents a frequency shift from the carrier. As can be seen, there are two lines; one is horizontal and the other is vertical, they are as follows:

The vertical line denotes the null guard band between the two coexisted systems and it equals to $\left(B W_{I}+B W_{V}\right) / 2 \mathrm{MHz}$. So, this line represents the overlapping and non overlapping frequency spectrum borders and divides the figure into two areas; the first is left from the line, it denotes the overlapping area. The two cases that follow this area are depicted in Figure 3 ( $a$ and $b$ ). The second area lies to the right of the vertical line; it represents the non overlapping area. The two cases that follow this area are depicted in Figure 3 ( $\mathrm{c}$ and $\mathrm{d}$ ).

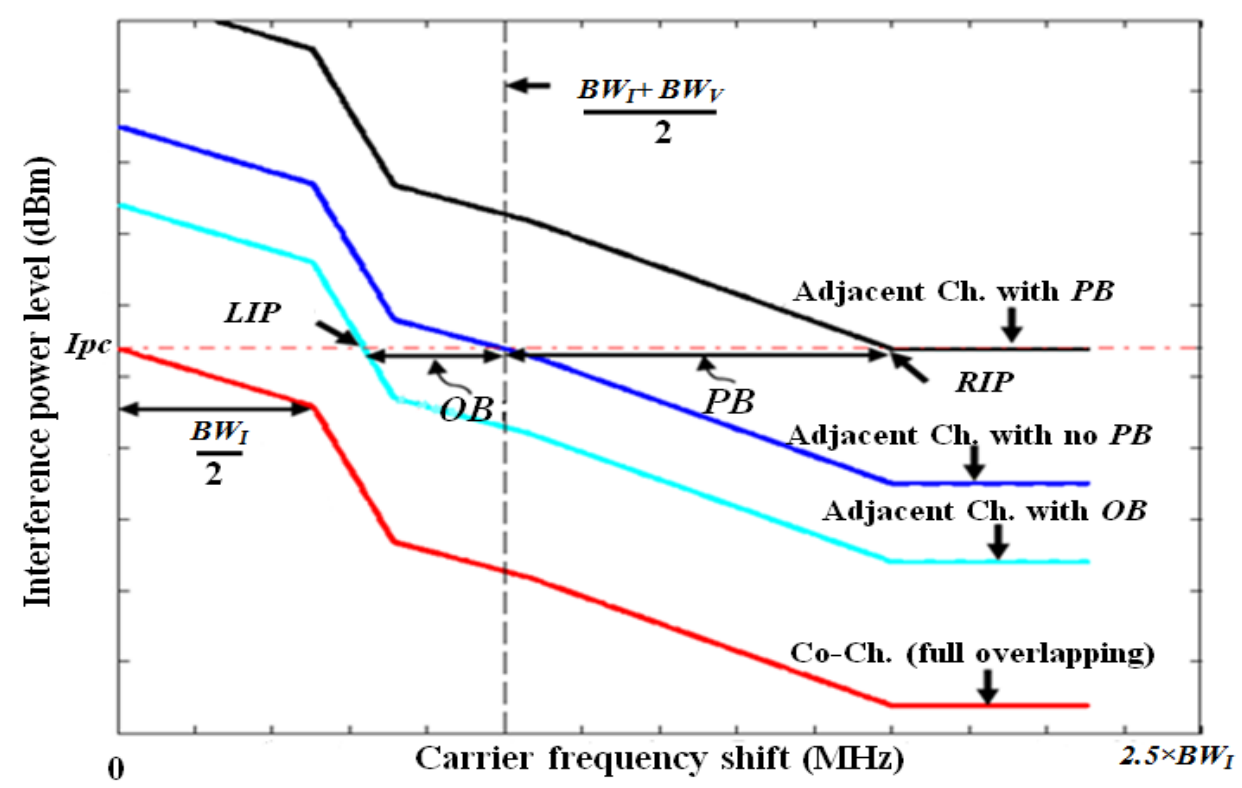

Figure 2. The Intersystem Interference Evaluation Scheme

a)

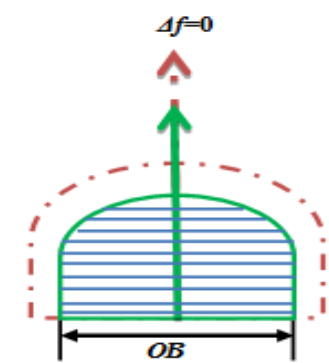

b)

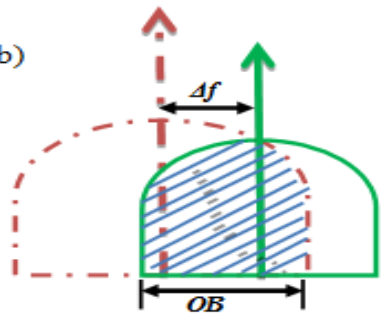

- . - . - . Interferer System c)

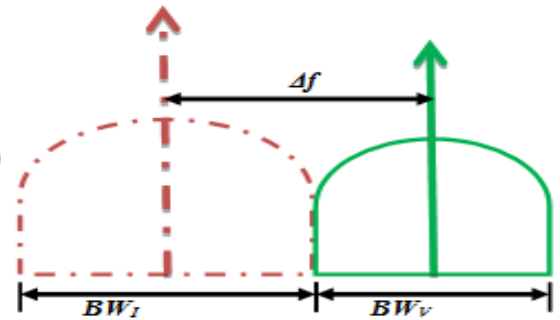

d)

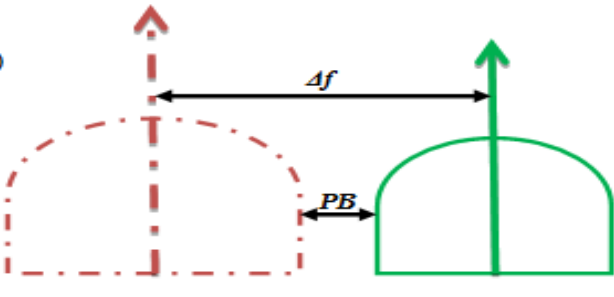

Interfered System

Figure 3. The Possible Intersystem Interference Scenarios: (a) Co-channel (Full Overlapping), (b) Adjacent Channel with an $O B$ (Partial Overlapping), (c) Adjacent Channel (with no $O B$ ) and (d) Adjacent Channel plus PB 
The horizontal line indicates to the interference protection criteria, $I_{p c}$, which equals, in this paper, to $-116 \mathrm{dBm}$. This line divides the figure into two areas and determines the coexistence situation; the curves in the upper area satisfy peaceful coexistence, whereas the curves under $I_{p c}$ line dissatisfy the coexistence conditions.

From Figure 2, it can be seen that, there are four possible coexistence scenarios, and all these scenarios must achieve the following formula:

$$
I(\Delta f)=I p c .
$$

The details of the possible scenarios are described in the following.

2.1.1. Co-Channel (Full Overlapping) Case: In this case, the carrier frequencies for both interferer and victim channels are typically identical and the amount of $\Delta f$ is $0 \mathrm{MHz}$. Such situation can happen when the interference level intersects the interference protection criteria, $I_{p c}$, at $0 \mathrm{MHz}$. In other words, the full overlapping scenario (co-channel) must follow equation (3) at

$$
\Delta f=0 \mathrm{MHz} .
$$

According to this, the size of overlapping band, $O B$, is maximum in this case and can be calculated from Figure 2 by equation (5).

$$
O B=\min \left\{B W_{I}, B W_{V}\right\}
$$

where $B W_{I}$ and $B W_{V}$ are the bandwidth of the interferer and victim systems, respectively. This case corresponds to a situation (a) in Figure 3.

2.1.2. Adjacent Channel Plus an $O B$ (Partial Overlapping) Case: This interference scenario take places once a portion of the interferer channel bandwidth overlaps with that of the victim system. The size of overlapping band, $O B$, can be less than $B W s / 2$, where BWs is the summation of the two system bandwidths. It is denoted by the dashed vertical line in Figure 2. Consequently, this scenario can be achieved if the interference power signal level intersects the interference protection criteria, $I_{p c}$, i.e., equation (3) should be realized at

$$
\mathrm{OMHz}<\Delta f<\frac{B W s}{2} \mathrm{MHz} .
$$

The size of $O B$ can be computed from Figure 2 by subtracting the frequency spectrum between $B W s / 2 \mathrm{MHz}$ point and the frequency offset at the left intersection point $\left(\Delta f_{L I P}\right)$, at which interference signal level crosses interference protection criteria line, $I_{p c}$, at any point to the left part of $B W s / 2$ point, i.e.,

$$
O B=\frac{B W S}{2}-\Delta f_{L I P}
$$

where $\mathrm{OMHz}<\Delta f_{R I P}<\frac{B W s}{2}$. This case corresponds to a situation (b) in Figure 3 .

2.1.3. Adjacent Channel (with no $\boldsymbol{O B}$ ) Case: For this scenario, the systems can operate peacefully concurrently by neighboring channels where no band is added in between. Such situation is achievable in line with equation (3) at

$$
\Delta f=\frac{B W s}{2} \mathrm{MHz} .
$$

This is graphically equivalent to the point at which three elements intersect together: $I(\Delta f), B W s / 2$ and $I_{p c}$. This case corresponds to a situation (c) in Figure 3.

2.1.4. Adjacent Channel Plus $P B$ Case: In some situations, frequency regulator bodies may obligate communication service suppliers to append a $P B$, to separate its frequency bands and other communication suppliers to avoid probable interference. Technically, this 
case is the relaxed one to initiate peacefully coexistence situation. But, it may be commercially the worse scenario due to the fact that it makes wasteful exploitation of available frequency resource. According to Figure 2 and equation (3), this case can happen for:

$$
\frac{B W s}{2} \mathrm{MHz}<\Delta f<\Delta f_{R I P} \mathrm{MHz}
$$

where $\Delta f_{R I P}$ is the right intersection point, RIP, where interference curve crosses the $I_{p c}$ value at the right lateral of $B W s / 2$ value. This situation is represented by Figure 3 (d). Consequently, the $P B$ can be computed, from Figure 2, by subtracting the frequency spectrum between $\Delta f_{R I P}$ and $B W s / 2 \mathrm{MHz}$, i.e.,

$$
P B=\Delta f_{R I P}-\frac{B W S}{2} .
$$

Where $\frac{B W s}{2}<\Delta f_{R I P} \leq 2.5 \times B W_{I}$.

\section{System Parameters and Assumptions}

The investigated coexistence scenario here considers that LTE-A base station (BS) is the interfering while the digital TV receiver is the victim. Although all other applicable interference scenarios can be investigated, but this scenario is only taken into account [16, 20] due to the fact that there is no real deterioration in the system performance (in average) if the interference scenario is between a system user (such LTE-A customer) and a different system user (TV receiver device). Additionally, the LTE-A BS emission to TV device situation is estimated as the crucial interoperability tricky because LTE-A BS is comparatively stationary and disturbs large numbers of TV receivers. Moreover, the power radiated from LTE-A BS is stronger than LTE-A customer.

In the assumed scenario, the receivers of TV are placed at different distances from LTE-A cell site coverage. In this situation, the physical separation between two systems is in the range 1-6 km as a macro radius in urban or suburban areas and it is greater than that for macro rural areas. The path loss channel propagation model in free space as well as the local clutter model are used in such coexistence cases as considered in [5] [19].

Tables 1, 2 and 3 list the intersystem interference factors for LTE-A system, TV digital receiver system, and channel model and coexistence parameters [5] [17] [21].

Table 1. LTE-A Base Station Parameters

\begin{tabular}{|l|l|}
\hline \multicolumn{1}{|c|}{ Parameter } & \multicolumn{1}{c|}{$\begin{array}{c}\text { Value } \\
\text { Cell radius }(\mathrm{km})\end{array}$} \\
\hline Width of channel $(\mathrm{MHz})$ & $\begin{array}{l}>6 \text { for macro rural scenario) } \\
\text { macro urban/suburban scenario) }\end{array}$ \\
\hline Effective isotropic radiated power $(\mathrm{dBm})$ & $5,10,20$ \\
\hline Transmitted power $(\mathrm{dBm})$ & 51 \\
\hline Antenna gain $(\mathrm{dBi})$ & 36 \\
\hline Antenna height $(\mathrm{m})$ & 15 \\
\hline Specral mask requirement & 30 \\
\hline
\end{tabular}


Table 2. TV Digital Receiver Parameters

\begin{tabular}{|l|c|}
\hline \multicolumn{1}{|c|}{ Parameter } & Value \\
\hline Width of channel $(\mathrm{MHz})$ & 8 \\
\hline Antenna height $(\mathrm{m})$ & 3 \\
\hline Antenna gain $(\mathrm{dB})$ & 12 \\
\hline receiver noise $(\mathrm{dB})$ & 5 \\
\hline receiver thermal-noise floor $(\mathrm{dBm})$ & -101 \\
\hline Permissible interference at the receiver $(\mathrm{dBm})$ & -116 \\
\hline
\end{tabular}

Table 3. Channel Model and Coexistence Parameters

\begin{tabular}{|l|l|}
\hline \multicolumn{1}{|c|}{ Parameter } & \multicolumn{1}{|c|}{ Value } \\
\hline Centre carrier freq. (MHz) & $800 \quad$ if $B W_{L T E-A}<B W_{T V}$ \\
\hline Correction bandwidth factor $(\mathrm{dB})$ & $0 \quad-10 \log \left(B W_{L T E-A} / B W_{T V-R}\right) \quad$ if $B W_{L T E-A} \geq B W_{T V}$ \\
\hline Channel and propagation model & Free space + clutter loss factor $(-40 \mathrm{~dB})($ ITU-R P.452-14) \\
\hline
\end{tabular}

\section{Results Analysis and Discussion}

In Table 4, the possible intersystem interference scenarios and compatibility factors required to fulfill coexistence between the two systems are listed (assuming no interference mitigation technique is used).

From Table 4, it is noted that full overlapping (co-channel) spectrum sharing scenario for the three channel bandwidths of LTE-A is much more difficult than the other scenarios. This is due to the long distance that is needed for achieving the compatibility. It recorded 375, 330 and $236 \mathrm{~km}$ for 5, 10 and $20 \mathrm{MHz}$ LTE-A, respectively. In the partial overlapping scenario, the required physical distance depends on the spectrum overlapping band size between the two systems, which in turn is based on the carrier frequency shift. For example, the required distance to achieve peaceful coexistence of $10 \mathrm{MHz}$ LTE-A with TV receiver, for an overlapping band of greater than $0 \mathrm{MHz}$ and less than $8 \mathrm{MHz}$, is less than $330 \mathrm{~km}$ and greater than $5.75 \mathrm{~km}$, correspondingly. In the case of no overlapping with a protection band, the minimum distance becomes more less than the other scenarios according to the size of $P B$.

In order to clarify the relation of physical path length between the two systems and the corresponding spectrum frequency shift under the assumption, Figure 4 is depicted. Figure 4 shows that as interferer channel bandwidth increases then both required physical path separation and frequency shift decrease. 


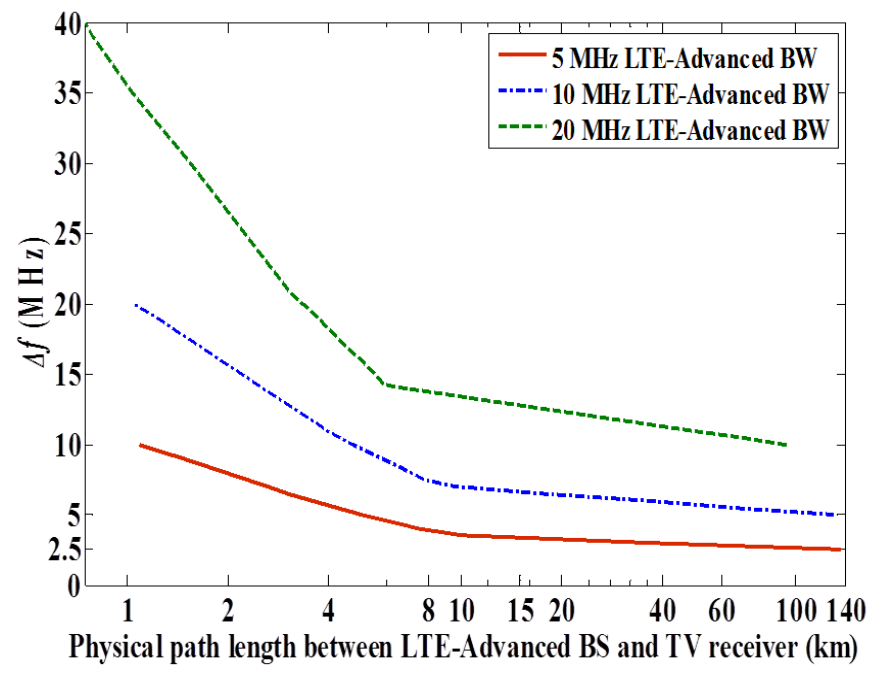

\section{Figure 4. Relationship of Separation Physical Path Length Between Systems, the Corresponding Frequency Span Required for Compatibility, and the Interferer System Bandwidth}

For peaceful operation of the two systems concurrently, the minimum frequency shift, $\Delta f$, should be no less than half of nominal BW of the interferer. For example, the spectrum frequency shift for LTE-A should be at least equal to or larger than 10, 5 and $2.5 \mathrm{MHz}$ for channel BW of system of 20, 10 and $5 \mathrm{MHz}$, correspondingly. Such result can be generalized for the adjacent channel with no $P B$ scenario. With the intention of coexist the two systems in full or partial overlapping scenario, the required distance is highly large, and may not be feasible, especially for short BS coverage, so an interference alleviation technique, such as antenna refinement should be employed to decrease the physical path length required. On the other side, the antenna refinement around the direction of signal path surrounds the leading interference signal coming from LTE-A into TV receiver. However, it is entirely not known which direction taken by the highest interference signal to reach the victim receiver antenna. This is important information as it shows that the antenna refinement cannot be necessarily reliable.

As shown in Table 4, different intersystem interference mechanisms are investigated under the antenna refinement loss effect to estimate the necessary separation distance to achieve peaceful coexistence between LTE-A and TV receiver systems. The size of frequency offset needed for the scenario of adjacent channel in case of $P B$ is zero, should be at least $6.5 \mathrm{MHz}, 9 \mathrm{MHz}$ and $14 \mathrm{MHz}$ for LTE-A system BW of $5 \mathrm{MHz}, 10 \mathrm{MHz}$ and $20 \mathrm{MHz}$, respectively. In other words, LTE-A and TV receiver systems can partially overlap if frequency shifts are not greater than $14 \mathrm{MHz}, 9 \mathrm{MHz}$ and $6.5 \mathrm{MHz}$ for the equivalent channel bandwidth of LTE-A as long as the obligatory additional isolation loss is fulfilled. For instance, in the case of $5 \mathrm{MHz}$ LTE-A and $5 \mathrm{~km}$ coverage area, a further attenuation of $30.9 \mathrm{~dB}$ is necessary for partial overlap of $4 \mathrm{MHz}$, whereas the overlapping size between the two systems reduces to $2.5 \mathrm{MHz}$ when isolation loss increases to 56.4 $\mathrm{dB}$.

When $5 \mathrm{MHz}$ LTE-A BS operates on co-channel frequency and covers an area of six kilometer, an extra attenuation of at least $62.9 \mathrm{~dB}$ to achieve compatibility with TV receiver with no harmful interference. However, the two systems have an opportunity to concurrently operate in adjacent frequency carrier and adding an amount of $P B$ between them.

For example, it is obligatory to put a $P B$ in the order of $3.5 \mathrm{MHz}$ (i.e., $\Delta f \geq 10 \mathrm{MHz}$ ) to get minimum isolation of no less than $12.9 \mathrm{~dB}$. Comparatively, Table 4 shows the significance of the propagation path loss effect on the required amount of $\Delta f$, and the 
necessary physical geographical isolation, namely, the involved isolation to manage compatibility of TV device and the LTE-A BS with a longer separation distance is smaller compared to the necessary isolation for a shorter separation distance which should be maximum.

Table 4. The Possible Intersystem Interference Scenarios and Compatibility Factors (NA: Not Applicable)

\begin{tabular}{|c|c|c|c|c|c|}
\hline $\begin{array}{l}\text { LTE-A } \\
\text { BW }\end{array}$ & $\begin{array}{l}\text { Coexistence } \\
\text { Factors }\end{array}$ & $\begin{array}{c}\text { Full } \\
\text { Overlaping } \\
(\text { co- } \\
\text { channel })\end{array}$ & $\begin{array}{c}\text { Partial } \\
\text { Overlapping }\end{array}$ & $\begin{array}{l}\text { No } \\
\text { Overlapping } \\
\text { with no } P B\end{array}$ & $\begin{array}{l}\text { No Overlap. with } \\
\qquad P B\end{array}$ \\
\hline \multirow{4}{*}{$5 \mathrm{MHz}$} & $\Delta f(\mathrm{MHz})$ & $\Delta f=0$ & $0<\Delta f<6.5$ & $\Delta f=6.5$ & $6.5<\Delta f \leq 12.5$ \\
\hline & $P B(\mathrm{MHz})$ & NA & NA & 0 & $0<P B \leq 6$ \\
\hline & $O B(\mathrm{MHz})$ & 5 & $0<O B<5$ & NA & NA \\
\hline & $\begin{array}{c}\text { Minimum } \\
\text { distance }(\mathrm{km})\end{array}$ & 375 & $3.05<$ dist. $<375$ & 3.05 & $1.09<$ dist. $\leq 3.05$ \\
\hline \multirow{4}{*}{$10 \mathrm{MHz}$} & $\Delta f(\mathrm{MHz})$ & $\Delta f=0$ & $0<\Delta f<9$ & $\Delta f=9$ & $9<\Delta f \leq 25$ \\
\hline & $P B(\mathrm{MHz})$ & NA & NA & 0 & $0<P B \leq 16$ \\
\hline & $O B(\mathrm{MHz})$ & 8 & $0<O B<8$ & NA & NA \\
\hline & $\begin{array}{c}\text { Minimum } \\
\text { distance }(\mathrm{km})\end{array}$ & 330 km & $5.75<$ dist. $<330$ & 5.75 & $0.054<$ dist. $\leq 5.75$ \\
\hline \multirow{4}{*}{$\begin{array}{c}20 \\
\mathrm{MHz}\end{array}$} & $\Delta f(\mathrm{MHz})$ & $\Delta f=0$ & $0<\Delta f<14$ & $\Delta f=14$ & $14<\Delta f \leq 50$ \\
\hline & $P B(\mathrm{MHz})$ & NA & NA & 0 & $0<P B \leq 36$ \\
\hline & $O B(\mathrm{MHz})$ & 8 & $0<O B<8$ & NA & NA \\
\hline & $\begin{array}{c}\text { Minimum } \\
\text { distance }(\mathrm{km})\end{array}$ & 236 & $6.76<$ dist. $<236$ & 6.76 & $0.75<$ dist. $\leq 6.76$ \\
\hline
\end{tabular}

Moreover, Figures 5-7 illustrate the relationship of attenuation resultant loss from antenna discrimination/ refinement with the needed geographical distance of between isolation losses required to accomplish the safe interoperability between the two considered systems, for various intersystem interference scenarios. These scenarios include co-channel (full overlapping) interference, partial overlapping by half of the smallest bandwidth of any of the two systems, $1^{\text {st }}, 2^{\text {nd }}$ and $3^{\text {rd }}$ adjacent channel interference (no guard band). For example, if $2 \mathrm{~km}$ separation distance is assumed between LTE-A (20 MHz) and TV receiver, then the required isolation should be 68.5, $60.5,43.9,30.1$ and $24.8 \mathrm{~dB}$ for co-channel (with $\Delta f$ of $0 \mathrm{MHz}$ ), partial overlapping of 4 $\mathrm{MHz}$ (with carrier frequency $\left(f_{c}\right)$ at $\triangle f=10 \mathrm{MHz}$ ), 1 st adjacent channel (with $P B$ of $0 \mathrm{MHz}$ and $f_{c}$ at $\Delta f=14 \mathrm{MHz}$ ), 2nd adjacent channel (with $P B$ of $8 \mathrm{MHz}$ and $\mathrm{f}_{\mathrm{c}}$ at $\Delta f=22 \mathrm{MHz}$ ) and 3 rd adjacent channel (with $P B$ of $16 \mathrm{MHz}$ and $f_{c}$ at $\triangle f=30 \mathrm{MHz}$ ), respectively. Also, it can be noticed that when geographical path between LTE-A BS and TV receiver becomes large needed attenuation dramatically diminishes especially for macro/rural area. 


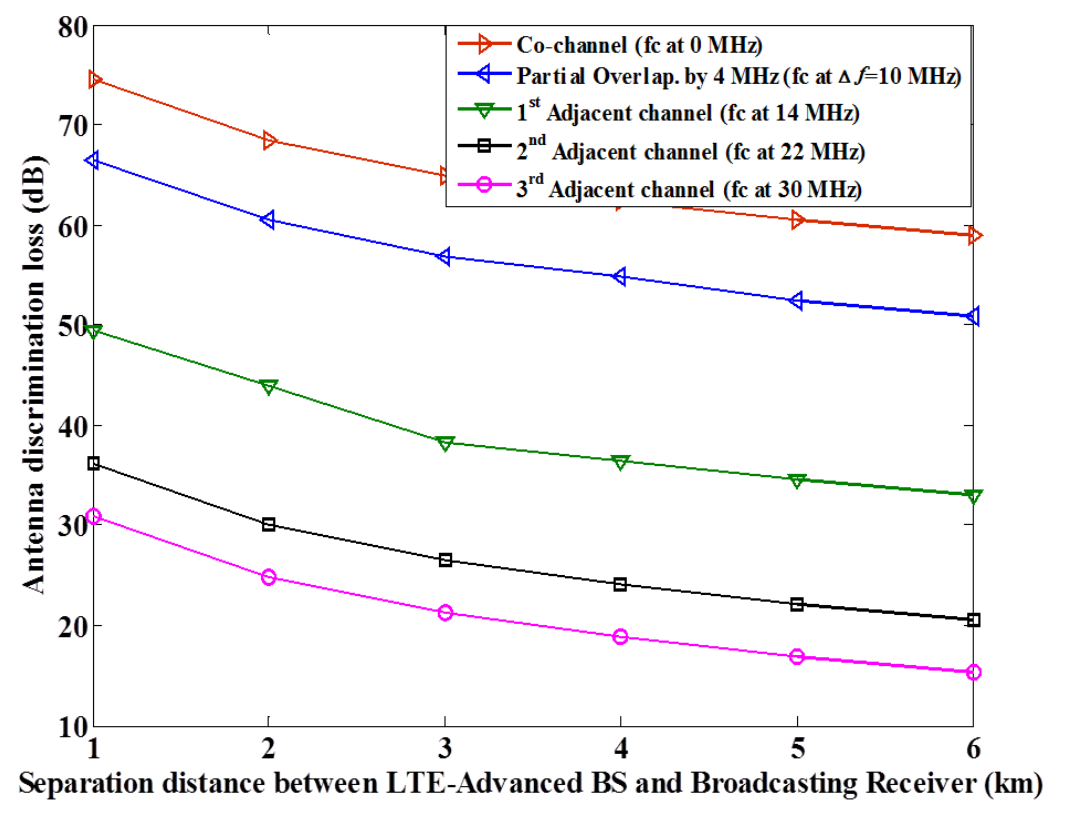

Figure 5. The Required Additional Isolation at Different Intersystem Interference Scenarios (LTE-A Works in $20 \mathrm{MHz}$ )

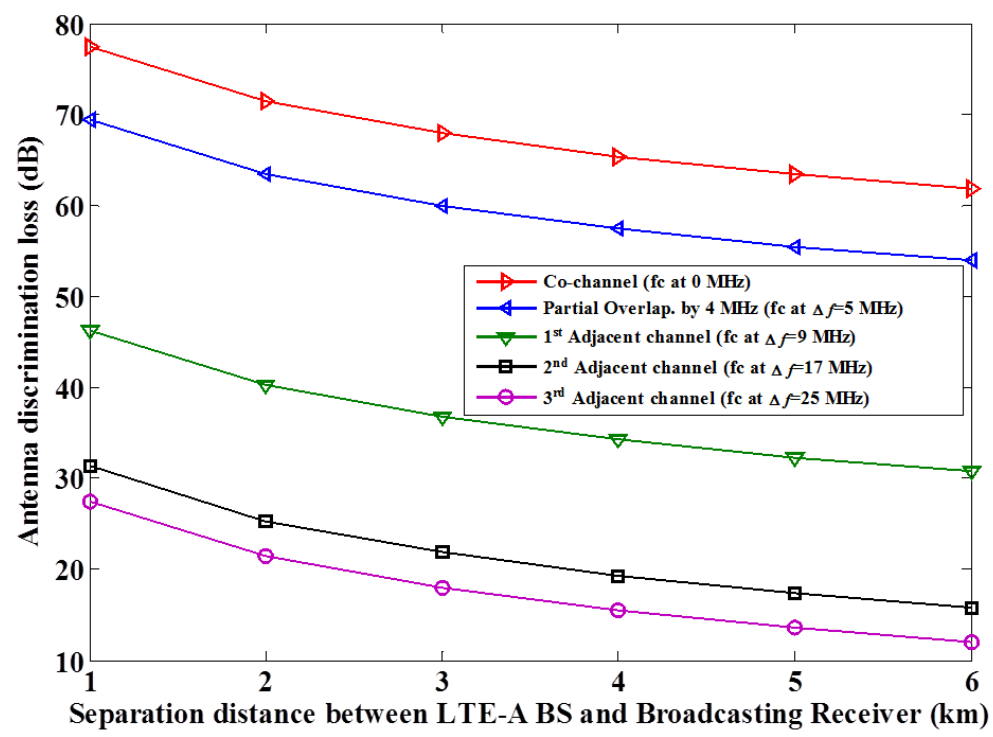

Figure 6. The Required Additional Isolation at Different Intersystem Interference Scenarios (LTE-A Works in $10 \mathrm{MHz}$ ) 


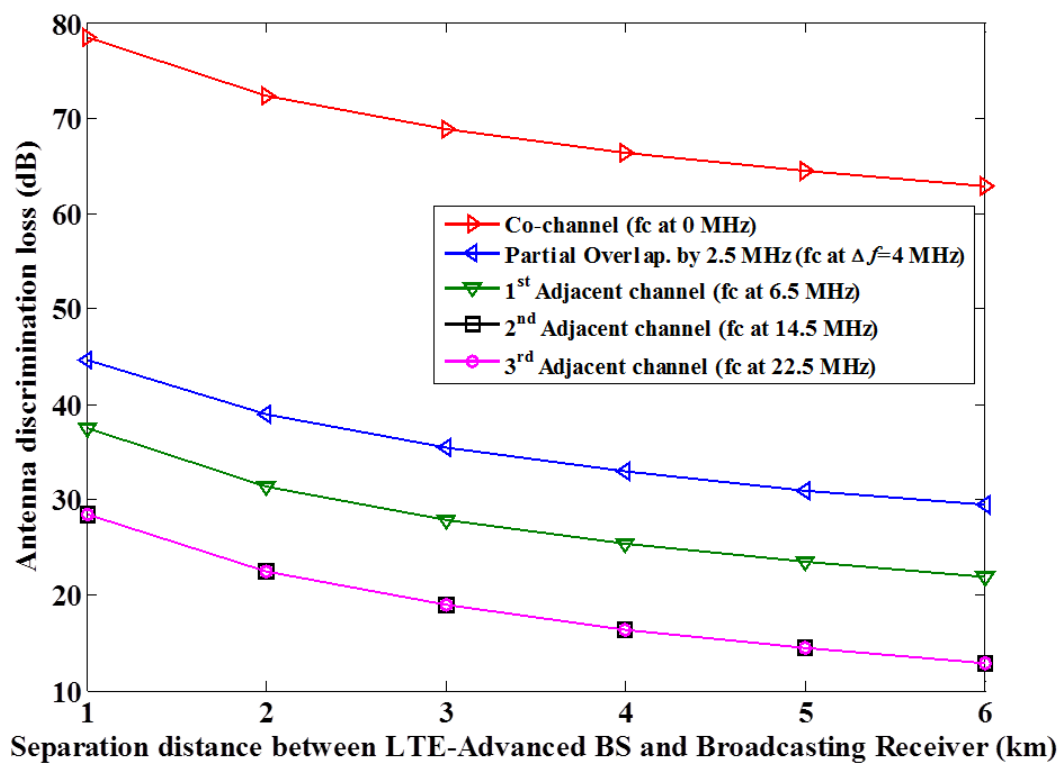

Figure 7. The Required Additional Isolation at Different Intersystem Interference Scenarios (LTE-A Works in 5 MHz)

Furthermore, by comparing Figures 5-7, it can be noted that co-channel interference (full overlapping) scenario in case of using $5 \mathrm{MHz}$ LTE-A is worse than both $10 \mathrm{MHz}$ and $20 \mathrm{MHz}$ LTE-A and the required antenna refinement loss is higher $(78.8 \mathrm{~dB})$ in case of 5 $\mathrm{MHz}$ LTE-A. On the other hand, Figures 5-7 show that $10 \mathrm{MHz}$ and $20 \mathrm{MHz}$ LTE-A scenarios behave better (less required additional losses: 77.5 and $74.5 \mathrm{~dB}$, respectively) than $5 \mathrm{MHz}$ LTE-A for co-channel interference (full overlapping) scenario. In addition, $10 \mathrm{MHz}$ and $20 \mathrm{MHz}$ LTE-A scenarios perform worse than $5 \mathrm{MHz}$ LTE-A scenario for partial overlapping and adjacent channel interference cases. This is due to the fact that, in $5 \mathrm{MHz}$ LTE-A and co-channel case, the entire channel bandwidth of LTE-A system is confined by digital broadcasting bandwidth $(8 \mathrm{MHz})$ and the signal power at partial overlapping and adjacent channel interference scenarios reduces more rapidly than that in case $10 \mathrm{MHz}$ and $20 \mathrm{MHz}$ LTE-A, and vice versa. Another reason for this result is the attenuation loss resulted in the correction band factor. This factor contributes with $1 \mathrm{~dB}$ to enhance coexistence in case of $10 \mathrm{MHz}$ LTE-A and $4 \mathrm{~dB}$ in case $20 \mathrm{MHz}$. However, in case of $5 \mathrm{MHz}$ LTE-A, bandwidth of LTE-A channel is narrower than that of TV receiver, thus the correction band factor effect is worthless.

Additionally, it can be seen in Figure 7 that the $2^{\text {nd }}$ and $3^{\text {rd }}$ adjacent channel curves are identical for same antenna discrimination loss and required separation distance. Such case may be due to the small frequency separation between the two systems compared to half of summation of the two system bandwidths $((5+8) / 2 \mathrm{MHz})$ when LTE-A BW is $5 \mathrm{MHz}$. Figure 7 also shows that when the interfered system bandwidth is smaller than that of the interferer, the $2^{\text {nd }}$ and above adjacent channels are identical even for very short separation distance.

\section{Conclusion}

A new graphically method has been proposed in this paper to study the interoperability between LTE-A downlink system and digital TV receiver. The findings indicate that the two systems undergo high interference in macro (urban/suburban/rural) areas when operating in co-channel scenario as a result of small geographical isolation separating the interfering and interfered systems. This situation shall impose on service providers to use a combination of strategies that improve the performance of the two systems, especially 
for spectrum sharing in very short distances (macro urban and suburban areas). The results revealed the importance of terrestrial separation distance and cellular category for spectrum sharing coordination, however, interference will be ineffective if the carrier frequency shift is managed to be at least half of the interferer bandwidth regardless the terrestrial category. LTE-A system as an interferer with wider channel bandwidth proved to be preferable than narrower bandwidth for peaceful compatibility, especially for cochannel scenario.

\section{References}

[1] 3GPP, "Technical Specification Group Radio Access Network; Further advancements for E-UTRA; LTE-A feasibility studies in RAN WG4 (Release 9)", (2010).

[2] S. Shah and U. Dalal, "Enhancing Capability of DVB-H by Three Layer Hierarchical Mapping", IETE Technical Review, vol. 31, no. 2, (2014), pp. 162-167.

[3] M. Khan and K. Han, "A Review of Handover Techniques in Wireless Ad hoc Networks Based on IEEE 802.21 Media Independent Handover Standard”, IETE Technical Review, vol. 31, no. 5, (2014), pp. 353-361.

[4] S. Ye, S.H. Wong, and C. Worrall, "Enhanced physical downlink control channel in LTE advanced Release 11", IEEE Communications Mgazine, vol. 51, no. 2, (2013), pp. 82-89.

[5] ITU-R P.452-14, "Prediction procedure for the evaluation of interference between stations on the surface of the Earth at frequencies above about $0.1 \mathrm{GHz}$ ", (2009).

[6] Z. A. Shamsan, T. A. Rahman, and A. M. Al-Hetar, "Interference coordination for LTE-A and FM broadcasting interoperability”, Annals of Telecommunications, vol. 67, (2012), pp. 477-483.

[7] W. A. Hassan, H.-S. Jo, M. Nekovee, C. Y. Leow, and T. A. Rahman, "Spectrum Sharing Method for Cognitive Radio in TV White Spaces: Enhancing Spectrum Sensing and Geolocation Database", KSII Trans. Internet and Information Systems, vol. 6, no. 8, (2012), pp. 1894-1912.

[8] M. Fuentes, C. Garcia-Pardo, E. Garro, D. Gomez-Barquero, and N. Cardona, "Coexistence of digital terrestrial television and next generation cellular networks in the $700 \mathrm{MHz}$ band", IEEE Wireless Communications, vol. 21, no. 6, (2014), pp. 63-69.

[9] M. Yang, Y. Li, D. Jin, L. Zeng, X. Wu, and A.V. Vasilakos, "Software-Defined and Virtualized Future Mobile and Wireless Networks: A Survey", Mobile Networks and Applications, vol. 20, no. 1, (2015), pp. 4-18.

[10] Y. He, J. Sun, X. Ma, A.V. Vasilakos, R. Yuan, and W. Gong "Semi-Random Backoff: Towards Resource Reservation for Channel Access in Wireless LANs", IEEE/ACM Transactions on Networking, vol. 21, no. 1, (2013), pp. 204-217.

[11] M. Youssef, M. Ibrahim, M. Abdelatif, L. Chen, and A.V. Vasilakos, "Routing Metrics of Cognitive Radio Networks: A Survey”, IEEE Communications Surveys and Tutorials, vol. 16, no. 1, (2014), pp. 92-109.

[12] H. Bawab, P. Mary, J.-F. Hélard, Y. Nasser, and O. Bazzi, "Global Ergodic Capacity Closed-Form Expression of Coexisting DVB-LTE-Like Systems," Proceedings of the 79th IEEE Vehicular Technology Conference (VTC Spring). (2014), pp. 1-5.

[13] L. Polak O. Kaller, L. Klozar, J. Sebesta, and T. Kratochvi, "Mobile Communication Networks and Digital Television Broadcasting Systems in the Same Frequency Bands: Advanced Co- Existence Scenarios", Radioengineering, vol. 23, no. 1, (2014), pp. 375-386.

[14] L. Polak, O. Kaller, L. Klozar and J. Prokopec, "Exploring and Measuring the Co-Existence between LTE and DVB-T2 Lite Services," Proceedings of the 36th International Conference on Telecommunications and Signal Processing (TSP 2013), (2013), pp. 316-320.

[15] L. Polak, L. Klozar, O. Kaller, J. Sebesta, M. Slanina and T. Kratochvil, "Study of Coexistence between Indoor LTE Femtocell and Outdoor-to-Indoor DVB-T2- Lite Reception in a Shared Frequency Band", EURASIP Journal on Wireless Communications and Networking. 2015 (1), (2015), pp. 1-14.

[16] W. A. Hassan, and T. A. Rahman, "A Spectrum Sharing Model for Compatibility between IMTAdvanced and Digital Broadcasting", KSII Trans. Internet and Information Systems, vol. 6, no. 9, (2012), pp. 2073-2085.

[17] Z. A. Shamsan, "LTE-advanced compatibility with digital broadcasting receiver at $800 \mathrm{MHz}$ ", Proceedings of the 2nd IEEE Saudi International Electronics, Communications and Electronics Conference (SIECPC'13), (2013), pp. 1-4.

[18] ITU-R BT.2265, "Guidelines for the assessment of interference into broadcasting service", (2014).

[19] CEPT REPORT 24. Technical Considerations Regarding Harmonization Options for the Digital Dividend. (2008),

[20] M. Mchenry, K. Steadman, and M. Lofquist, "Determination of Detection Thresholds to Allow Safe Operation of Television Band "White Space" Devices", Proceedings of the 3rd IEEE Symposium on New Frontiers in Dynamic Spectrum Access Networks (DySPAN ‘08), (2008), pp. 1-12. 
[21] E. Dahlman, S. Parkvall, and J. Skold, 4G: LTE/LTE-A for Mobile Broadband, Academic Press. (2011), pp. 347-377.

\section{Author}

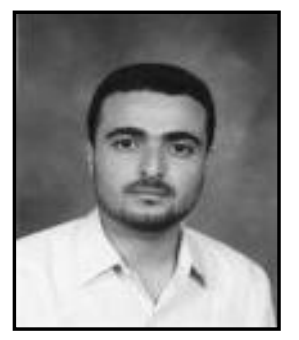

Zaid A. Shamsan, he received the B.Eng. (Hons.) degree in electronics and communication engineering from Sudan University of Sciences and Technology, Sudan, in 2002, and the M.Eng. and Ph.D. degrees in Telecommunication and Electrical Engineering from Universiti Teknologi Malaysia (UTM), Malaysia, in 2007 and 2010, respectively. He was a Postdoctoral Research Fellow in Wireless Communication Centre at UTM, from 2010-2012. Dr. Shamsan is currently an Assistant Professor with Faculty of Electrical Engineering, Al Imam Mohammad bin Saud Islamic University, Saudi Arabia. Since 2003, he has been an academic staff in the Faculty of Engineering and Information Technology, Taiz University, Yemen. He has published more than 45 scientific papers in high impact factor and archival technical journals and conferences. His research interests include orthogonal frequency-division multiplexing technology, interference analysis techniques, wave propagation, mathematical modeling for coexistence analysis in wireless networks, rain fading analysis and mitigation, free-space optics, and optical communication. 
International Journal of Future Generation Communication and Networking Vol. 9, No.9, (2016) 